\title{
THE STRUCTURE OF NORMED ABELIAN RINGS
}

\author{
EDGAR R. LORCH
}

Introduction. A very prominent feature of the development of the theory of function spaces, a branch of mathematics which deservedly or not has attracted very concentrated attention since the turn of the century, is that one of the essential and defining features of a function was rapidly eliminated from attention. The property to which we refer is the ring property, more properly the multiplicative property, which demands that the multiplication of any two elements be allowed. Thus when the study of these spaces was sufficiently developed to be cast into abstract form, the basic domain of operations was not a ring but merely a group, an additive group or module, with real or complex operators. When a suitable topology is introduced into such a group it becomes a space. The spaces $\mathfrak{B}$ defined by Banach seem to merit the most attention. The topology introduced is of the simplest character: a metric, invariant under translation, and homogeneous with respect to scalar (that is, real or complex numerical) multiplication. The elements of these metric groups are called vectors.

It is only very recently that its birthright has been restored to this theory and that function spaces are being studied as rings and not merely as groups. The present address will attempt to delineate some aspects of the recent developments, to point to certain achievements, and to suggest some problems. The compilation of problems, the formulation of open questions, is usually a hazardous matter. It is frequently difficult to understand the importance of a question before it has been answered. Thus our suggestions will be largely tentative.

Definition and examples of normed rings. To begin with, a Banach space is an additive group of elements $a, b, c, f, g, \cdots$ with operators $\alpha, \beta, \lambda, \mu, \cdots$ which are complex numbers (in the present work real scalars will be excluded). Every element $a$ has a norm $|a|$ (also written $\|a\|$ or even $\|\mid a\| !$ ) which is a non-negative number. The space is metrized by the norm with dist $(a, b)=|b-a|$; it is complete in this norm. Finally the norm is homogeneous, that is, $|\alpha a|=|\alpha| \cdot|a|$. We are now in a position to define a normed abelian ring.

An address delivered before the New York meeting of the Society on April 29, 1944, by invitation of the Program Committee; received by the editors May 2, 1944. 

ring if

Definition. $A$ collection $\Re$ of elements is called a normed abelian

(i) $\Re$ is a Banach space.

(ii) $\Re$ is a ring with unit ${ }^{1}$ e. Multiplication satisfies the axiom $a b=b a$ (abelian character).

(iii) Under multiplication, the norm is subject to the inequality

$$
|a \cdot b| \leqq|a| \cdot|b| \text {. }
$$

These axioms were first given by Nagumo in $1936 .{ }^{2}$ To indicate the versatility of the concept here defined some examples will be cited. At the head of the list will be placed certain classic rings of analysis.

1. The ring of complex numbers.

2. The ring of complex valued functions $f(x)$ bounded and continuous on a metric space $S$ with $|f|=1$.u.b.s $|f(x)|$ (for example, $S$ might be the interval $0 \leqq x \leqq 1$ ).

3. The ring of functions $f(z)$ analytic over some bounded domain $D$ and continuous over the closure $\bar{D}$ of $D$ with $|f|=1$.u.b. $\bar{D}|f(z)|$.

4. The ring of complex valued functions $f(x)$ of bounded variation on an interval $a \leqq x \leqq b$ with $|f|=$ l.u.b. $[a, b]|f(x)|+V_{[a, b]} f(x)$.

5. The ring of absolutely convergent Fourier series $f(x)=\sum_{-\infty}^{\infty} a_{n} e^{i n x}$ with $|f|=\sum_{-\infty}^{\infty}\left|a_{n}\right|$.

6. The ring of operators $\phi(A)$ defined by means of functions $\phi(\lambda)$ which are measurable and bounded with respect to a resolution of the identity $E(\lambda),-\infty<\lambda<\infty$, in a reflexive vector space.

7. The ring generated by any bounded linear operator $T$ defined over an arbitrary Banach space $\mathfrak{B}$ (or ring $\mathfrak{R}$ ) with $|T|=$ l.u.b. $|T f|$, $|f|=1, f \in \mathfrak{B}$. The ring then consists of the polynomials in $T$ with their limits in the indicated topology (uniform topology).

8. The ring generated by any one-parameter group or semi-group of linear transformations in a Banach space.

9. The ring of complex valued functions $f(x), a \leqq x \leqq b$, possessing $m$ continuous derivatives with $|f|=\sum_{n=0}^{m}(n !)^{-1}$ l.u.b. $[a, b]\left|f^{(n)}(x)\right|$.

10. The ring $\Re$ of complex valued functions $f(x), a \leqq x \leqq b$, possessing infinitely many derivatives in which the norm is introduced as follows: Let $M_{n}>0, n=0,1,2, \cdots$, be a sequence of constants with $M_{n} /\left(M_{r} \cdot M_{n-r}\right) \geqq n ! /[r !(n-r) !], r=0,1, \cdots, n$. Then $f(x) \in \Re$ if $\sum_{n=0}^{\infty}\left(M_{n}\right)^{-1}$ l.u.b. $[a, b]\left|f^{(n)}(x)\right|<\infty$. This series serves as the norm of $f(x)$.

1 There is little point in investigating rings without unit. But if $R$ has no unit, it can be embedded in a normed ring $R_{1}$ with unit and such that $R$ is a maximal ideal in $R_{1}$.

${ }^{2} \mathrm{M}$. Nagumo, Einige analytische Untersuchungen in linearen metrischen Ringen, Jap. J. Math. vol. 13 (1936) pp. 61-80. 
At this point a few bibliographical remarks seem pertinent. Example 1 is the core of every ring. It will be seen shortly how the complex numbers can be distinguished from other rings. Rings in 6 were first considered by v. Neumann and Stone for Hilbert space. ${ }^{3}$ The author subsequently showed that all the essential phenomena could be reproduced in a Banach space providing it was reflexive. ${ }^{4}$ Since then this particular problem has been considered by $F$. Riesz who founds his discussion not on normed rings but partially ordered spaces. ${ }^{5}$ Example 7 is particularly important in the present discussion. It will be seen later in our treatment of maximal ideals that a ring 7 is locally homomorphic to rings of type 2 or 3 or 9 or 10 . The ring 7 where $T$ is a bounded self-adjoint transformation in Hilbert space has a structure which permits with ease the finding of the resolution of the identity of $T$ (which, incidentally, is not usually in $\Re$ ). This will be shown below. Although the determination of the structure of transformations by operating with their rings (rather than with the clumsier spaces over which they are defined) has had very signal successes, there are some points at which this approach has not yet yielded ground. An ideal-theoretic procedure for determining in 8 the resolution of the identity of a one-parameter strongly continuous group $U_{0}$ of unitary transformations on Hilbert space has failed up to this moment. ${ }^{6} \mathrm{~A}$ fortiori, for the case of rings 8 generated by semi-groups in Banach spaces attempts by the author to extend the results of Hille $^{7}$ have not been successful. The rings 10 are similar to those treated in the literature. ${ }^{8}$ Incidentally, as an example of constants $M_{n}$ satisfying the given conditions one may take $M_{n}=(n !)^{8}, s \geqq 1$. If $s=1$ the ring contains exclusively analytic functions. The results of Gelfand relating to ring 5 are well known to analysts. However the parent theory of which these results on absolutely convergent

${ }^{3} \mathrm{~J}$. v. Neumann, Über Funktionen von Funktionaloperatoren. Ann. of Math. (2) vol. 32 (1931) pp. 191-226; M. H. Stone, Linear transformations in Hilbert space, Amer. Math. Soc. Colloquium Publications, vol. 15, 1932, chap. 6.

- E. R. Lorch, On a calculus of operators in reflexive vector spaces, Trans. Amer. Math. Soc. vol. 45 (1939) pp. 217-234.

$\checkmark \mathrm{F}$. Riesz. Sur quelques notions fondamentales dans la théorie générale des opérations linéaires, Ann. of Math. (2) vol. 41 (1940) pp. 174-206.

- This is a theorem due to Stone to the effect that $U_{s}=\exp (i s H), H$ self-adjoint. The fact that $H$ may be unbounded causes the difficulty.

7 E. Hille, Notes on linear transformations. II. Analyticity of semi-groups, Ann. of Math. (2) vol. 40 (1939) pp. 1-47. See also more recent announcements in the Proc. Nat. Acad. Sci. U.S.A. vol. 28 (1942) pp. 175-178 and 421-424; vol. 30 (1944) pp. 58-60.

See for instance S. Mandelbrojt, Analytic functions and classes of infinitely differentiable functions, The Rice Institute Pamphlet vol. 29 (1942) No. 1. 
Fourier series are merely a biproduct is not so widely appreciated. ${ }^{\ominus}$

Reducibility of rings. In the ring $\Re$, every element $a$ gives rise to an operator $T_{a}$ by virtue of the correspondence $x \rightarrow T_{a} x=a x, x$ in $\Re$. The operator $T_{a}$ is linear and bounded with $\left|T_{a}\right| \leqq|a|$. This gives a representation of $\Re$ as a ring of operators; it is the regular representation. By introducing a new norm in $\Re$ equivalent to the old, we have $\left|T_{a}\right|=|a|$. We shall assume that this has been done. This change is not significant as almost all the results which we give later are invariant under algebraic and topological isomorphisms. Thus we see that the theory of linear transformations may be applied to determine the structure of the ring. The complex numbers $\lambda$ for which $(a-\lambda e)^{-1}$ does not exist constitute the spectrum of $a$. On the other hand, the properties of linear transformations may be studied in very large part in rings. For if $T$ is a linear transformation over a space $\mathfrak{B}, T$ can be embedded in a ring $\Re$ in which it has the same spectrum as over $\mathfrak{B}$. The union of the concepts and methods of linear transformations and rings produces a numerous and healthy progeny.

The fundamental formula for studying the reduciblity of a ring $\Re$ is the Cauchy integral

$$
j=\frac{1}{2 \pi i} \int_{c} \frac{d \zeta}{\zeta e-a},
$$

where $a$ is any fixed element and $C$ is any simple rectifiable curve lying in the complex plane and avoiding entirely the spectrum of $a$. It is easily shown that $j^{2}=j$. If for some $a$ and $C, j \neq e$ or 0 , and only then, will $\Re$ be reducible. An integral of the type (2) expressed for operators and in slightly different notation was given many years ago by $\mathrm{F}$. Riesz in his book. ${ }^{10}$ Riesz mentions very briefly the elementary properties of $j$. The results of Riesz seem to have been forgotten in the last decade; there is no mention of them in the literature, particularly that literature which could have employed them to considerable advantage. The present author rediscovered (2) and elaborated certain of its consequences with some completeness. ${ }^{11}$ This particular Cauchy integral is a powerful tool and may serve to shorten very considerably solutions which have recently appeared. We shall state

๑ I. Gelfand, Normierte Ringe, Rec. Math. (Mat. Sbornik) N.S. vol. 9 (1941) pp. 1-24. The applications of the abstract theory to absolutely convergent series is found in the same volume: Über absolut konvergente trigonometrische Reihen und Integrale, pp. 51-66.

${ }^{10} \mathrm{~F}$. Riesz, Les systèmes d'equations linéaires, Paris, 1913, pp. 117-121.

$11 \mathrm{E}$. R. Lorch, The spectrum of linear transformations, Trans. Amer. Math. Soc. vol. 52 (1942) pp. 238-248. 
the facts on the reducibility of $R$ in the form of a theorem. ${ }^{12}$

THEOREM 1. The ring $\Re$ is irreducible if and only if the spectrum of every element $a$ in $\Re$ is a connected set.

If $\Re$ is reducible, it is the direct sum of two rings, $\Re=\Re_{1}+\Re_{2}$. Furthermore $\Re_{1}$ is isomorphic to the quotient ring $\Re / \Re_{2}$. Thus the study of reducibility is at its most demanding level the study of ring homomorphisms. The general homomorphisms will claim our attention later.

If $\Re$ is irreducible it may under proper circumstances be embedded in a ring $\Re^{\prime}$ where that property abounds. This may be essayed in case $\Re$ is a ring of linear transformations defined over a Banach space $\mathfrak{B}$. Then $\mathfrak{R}^{\prime}$ is taken to be the ring of bounded operators over $\mathfrak{B}$ which are limits of operators in $\Re$ in some topology weaker than the uniform-for example, the strong or the weak topology. This procedure is that which has been used to obtain the structure of a selfadjoint transformation in Hilbert space or of a one-parameter group of unitary transformations, also in Hilbert space.

Maximal ideals. The importance of the contributions of Gelfand in the theory of normed rings arises from the fact that he first considered the ideals of such rings. ${ }^{18} \mathrm{His}$ results deal almost exclusively with maximal ideals. An ideal $\Im$ is a subset of $\Re$ closed in the usual algebraic sense and in the topological sense as well. A maximal ideal is one distinct from $\Re$ which has no proper extensions. The existence of maximal ideals may be established easily by the use of Zorn's lemma. Every ideal $\Im$ is embedded in one or more maximal ideals. The maximal ideals are characterized by the fact that their residue classes contain precisely one scalar $\lambda e$. Thus if $x \in \Re$ then $x \equiv \lambda e(\Im)$ where $\Im$ is maximal. This gives rise to complex valued functions $x(\Im)=\lambda$ defined over the class of maximal ideals. Gelfand introduces a topology (in fact, more than one topology is used) in the class $\mathfrak{M}$ of maximal ideals in a standard fashion so that $\mathfrak{M}$ becomes a bicompact Hausdorff space. Over this space, the functions $x(\Im)$ are constrained to be continuous. Thus the following theorem is obtained. ${ }^{14}$

THEOREM 2. The mapping $x \rightarrow x(\Im)$ is a homomorphism which maps

$12 \mathrm{E}$. R. Lorch, The theory of analytic functions in normed abelian vector rings, Trans. Amer. Math. Soc. vol. 54 (1943) pp. 414-425; in particular, Theorem 2. The more important problems dealt with in this paper are treated in the first half of the present address.

${ }^{13}$ See footnote 9.

14 Normierte Ringe, Theorem 10. 
the ring $\Re$ onto a subring of the ring of functions continuous on the set $\mathfrak{M}$ of maximal ideals in $\Re$.

Two facts should be noted. First, the ideal $\Omega$ which maps into the function which is identically zero is the radical of $\Re$ (see below). Secondly, assuming that the radical is empty or has been factored out, the situation is far from clear. The ring of functions $x(\Im)$ is a subring of the ring of continuous functions on $\mathfrak{M}$ and in this subring the norm is not (necessarily) the norm of uniform approximation. Thus this theorem gives an initial but not definitive result on the structure of $\Re$. More penetrating results of this type will be considered in the second half of this address.

The operation of forming factor rings $\Re / \Im$ will be performed frequently. The topology is introduced into $\bar{\Re}=\Re / \Im$ as follows: If $a \in \bar{\Re}$ is the image of $a$ in $\Re$ then $|\bar{a}|=$ g.l.b. $|a+q|$ where $q$ ranges over $\Im$. With this norm $\bar{\Re}$ is a (complete) normed ring.

The radical. A nilpotent is an element $a$ in $\Re$ such that for some positive integer $n, a^{n}=0$. To define the radical of $\Re$ it is necessary to introduce the notion of quasi-nilpotent. A quasi-nilpotent is an element $a$ such that for every complex number $\mu, \lim _{n \rightarrow \infty}(\mu a)^{n}=0$. The structure of the radical has been given by Gelfand..$^{15}$ It can equally well be determined from a lemma used by the author to determine the structure of transformations. ${ }^{16}$ The portion of the lemma we shall need follows.

Lemma. Let the curve $C$ in formula (2) be the unit circle. Then $j$ in (2) satisfies the equation $j=\lim _{n \rightarrow \infty}\left(e-a^{n}\right)^{-1}$. Furthermore $j b=b$ if and only if $\lim _{n \rightarrow \infty} a^{n} b=0$.

Suppose that $a$ has only the singularity $\lambda=0$. Then the same is true for $(\mu a)^{n}$. The integral (2) applied to $\mu a$ with $C$ the unit circle gives $j=e$. Applying the lemma, $j e=e$ implies that $\lim _{n \rightarrow \infty}(\mu a)^{n}=0$. Conversely, if $\lim _{n \rightarrow \infty}(\mu a)^{n}=0$, then for all $\mu$, the spectrum of $\mu a$ lies in the unit circle, hence consists of the one point $\lambda=0$.

THEOREM 3. The quasi-nilpotents in $\Re$ are those elements a whose spectrum is the one point $\lambda=0$.

Applying the theory of maximal ideals it is clear that the quasinilpotents constitute an ideal, the radical $\Omega$ of $\Re$. The radical is the intersection of all maximal ideals. Obviously, the ring $\Re / \Omega$ is without radical. It is this ring $\Re / \Re$ which according to the previous section is

${ }^{15}$ See footnote 9.

${ }^{16}$ See footnote 11, Theorem 7. 
isomorphic to a function ring. For if $x$ is in $\Re$, then $x(\Im)=0$ for all maximal ideals $\Im$.

Since the spectrum of the radical is concentrated at one point all methods for cracking the spectrum into pieces, in particular, methods applicable to connected spectra, are naturally of little value. The structure of the radical is largely unknown. In fact the class of examples of radicals is as yet quite limited.

Rings which are fields. A very vital question to all of mathematics is the determination of whether there exist normed fields which are extensions of the complex field. This problem was settled by Mazur in $1938^{17}$ by proving that no norm could be introduced in the field of rational functions with complex coefficients. Mazur also established the fact that if in a normed ring we have instead of the inequality (1) the equality $|a \cdot b|=|a| \cdot|b|$, then $\Re$ is the field of complex numbers. Both of these results seem to be most fittingly proved by the methods of the present theory. To establish the first, use is made of the fact that the spectrum of an element is not empty. This was proved for linear transformations by A. E. Taylor. ${ }^{18}$ Armed with this theorem, the proof is completely trivial. The second result is obtained by an appropriate application of our lemma and some associated properties of linear transformations. ${ }^{19}$

Analytic functions in $\Re$. It was noted very early in the development of linear operations that the notion of analytic function was destined to play here a very considerable role. Probably the first example of such a function was given by the equation for the resolvent $(e-a)^{-1}=e+a+a^{2}+\cdots$ which is valid for $|a|<1$. An important consequence of this equation is that the regular elements in $\Re$ (that is, those elements which have an inverse-also called the nonsingular elements) form an open set. A very large literature has been created about general analytic function theory. It will be impossible to give here an account of it which is in any sense adequate. However, in order to outline the principal results of the first part of this paper it will be necessary to touch on and examine briefly some of the points of view which have been advanced. It is hoped that the brevity of our discussion will not produce a distortion of the situation.

A point of view frequently employed is that which conceives analytic functions as defined over the complex plane and having values which lie in some Banach space. Such a view does not allow one to

${ }^{17}$ S. Mazur, C. R. Acad. Sci. Paris vol. 207 (1938) pp. 1025-1027.

${ }_{18}$ A. E. Taylor, The resolvent of a closed transformation, Bull. Amer. Math. Soc. vol. 44 (1938) pp. 70-74.

${ }^{19}$ See footnote 11 , Theorem 8. 
realize the product of two functions. Another view considers functions once more defined for complex numbers and whose values are operators over some Banach space. Here the operation of product of analytic function is permissible but that of forming a function of a function is inadmissible. Although these and still other approaches have "generality," it is still to be decided by each individual worker in this field whether they deserve to be considered as legitimate extensions of the theory of analytic functions. Our own decision is adverse.

A solution to the problem of extending suitably the scope of analytic function theory was presented to this Society by the author slightly over two years ago. It will be described briefly. The domain over which this theory is developed is any normed abelian ring $\Re$. The functions $f(z)$ treated here are assumed to be defined over a region in $\Re$ and to have their values in $\Re$. It is rather remarkable that the development of the theory parallels very closely the classic course in its methods and principal identities. Naturally there are points which require considerable care in the case of rings which for the case of the complex plane are non-existent or trivial. Starting with a definition of derivative couched in the form

$$
\left|f\left(z_{0}+h\right)-f\left(z_{0}\right)-h f^{\prime}\left(z_{0}\right)\right|<\epsilon|h|
$$

(for $|h|<\delta$, and so on) the Cauchy theorem is rapidly obtained for regions convex in $\Re$ or those which are the sum of finitely many such. The general region in $\Re$ is of a complicated topological structure and the Cauchy theorem has not been investigated for it. What is here required is that the "interior" of a curve lie inside the region. This will be touched upon later.

In the next place the Cauchy formula is established. Once more the choice of the curve $C$ is critical. For example the formula (2) yields not merely the values 0 and $e$ but all other idempotents $j$. A whole class of complications of this sort may be avoided at the outset by assuming that $\Re$ is irreducible. The cycle of fundamental theorems is completed with the development of an analytic function into a power series. This series converges in the largest sphere with center at the point of development in which the function is analytic. It may converge for points outside this sphere. For example, any series converges for all points in the radical.

In the development of the theory of analytic functions up to the present point, the ideals in $\Re$ have played no role. There is a class of ideas in which the mixture of the two concepts is fruitful. We shall give one application of this procedure. The proof is based on the 
Taylor series expansion for these functions.

THEOREM 4. If $f(z)$ is a function analytic in a region $D$ in $\Re$ and $\Im$ is any ideal, then $\bar{f}(z)$ is analytic in the region $\bar{D}$ of the ring $\Re / \Im$. Here $\bar{f}(z)$ and $\bar{D}$ represent the images of $f(z)$ and $D$ under the homomorphism $\Re \rightarrow \Re / \Im$.

It should be noted that, under a homomorphism, a sphere in $\Re$ is mapped into a sphere in $\bar{\Re}$ of the same radius. Thus sphere of convergence goes into a subsphere of the sphere of convergence. If the ideal $\Im$ of the theorem is a maximal ideal, then $\vec{f}(z)$ is a function analytic in the classic sense since $\Re / \Im$ is the field of complex numbers. Thus we see that the ring of functions analytic over a certain domain in $\Re$ may be mapped homomorphically on a certain ring of functions analytic in the complex plane. The set of functions analytic in a domain of $\Re$ is considerably extensive; it includes as a proper subset all functions analytic in the ordinary sense (yet considered as generating functions in $\Re$ ). The above theorem is merely one of a host of homomorphism theorems which may be developed in this field. If the homomorphism proceeds in the direction: function of a complex number to function in a general ring, $f(\lambda) \rightarrow f(a)$, it is commonly referred to as an operational calculus. As we shall meet more homomorphism theorems later it is worth noting the specific hypotheses which support them.

A further homomorphism may be indicated at this point since it has received attention in the literature. Suppose $C$ is an arbitrary rectifiable arc in $\Re$, closed or not, $\phi(\zeta)$ is a function continuous on $C$ and $a$ is an element in $\Re$ for which $(\zeta-a)^{-1}$ exists, $\zeta$ on $C$. Then $(\zeta-z)^{-1}$ exists for $z$ in a certain neighborhood of $a$ and

$$
f(z)=\frac{1}{2 \pi i} \int_{c} \frac{\phi(\zeta)}{\zeta-z} d \zeta
$$

is easily shown to be analytic in this neighborhood. If now $C$ is taken to be a simple closed curve in the complex plane, $z$ is held fast at the value $a$ where $a$ has its spectrum entirely within $C$ and if $\phi(\zeta)$ is a function of the complex variable $\zeta$ analytic in the classic sense in a region containing $C$ and its interior then we may say that $\phi(\zeta)$ in (4) defines the element $\phi(a)$ in $\Re$ (instead of $f(z)$ or $f(a)$ ). This gives an operational calculus $\phi(\lambda) \rightarrow \phi(a)$ which has been treated by Gelfand, Dunford, and A. E. Taylor. ${ }^{20}$ The element $\phi(a)$ is termed an "ana-

${ }^{20}$ Gelfand, loc. cit. Theorem 19; N. Dunford, Spectral theory, Bull. Amer. Math. Soc., vol. 49 (1943) pp. 637-651, in particular p. 641; A. E. Taylor, Analysis in complex Banach spaces, Bull. Amer. Math. Soc. vol. 49 (1943) pp. 652-669, Theorem 9. 
lytic function of $a . " 21$ Certain important functions of $a$ are excluded by this device. Suppose for instance that the spectrum of $a$ is the circle $|\lambda|=1$. Then since $\log \zeta$ is not analytic in any region containing this circle, $\log a$ is not produced by this operational calculus. However the function $\log z$ may well be defined for $z=a$. An explanation for this phenomenon lies in the fact that $\log a$ does not exist in the subring of $\Re$ generated by $a$ since the spectrum of $a$ in the smaller ring consists of the solid circle $|\lambda| \leqq 1$.

Up to the present moment there is little known of the character of the domains over which analytic functions are defined and of the Riemann surfaces associated with them. An example will indicate some of the phenomena which may be expected. The regular elements $a$ in $\Re$ (those for which $a^{-1}$ exists) form a topological group. This group consists of one or more maximal connected open components or domains. ${ }^{22}$ If the number is more than one, it may be shown that it is infinite. The component containing $e$ is called the principal component. The function $\log z=\int_{b}^{z} \zeta^{-1} d \zeta$ is defined precisely for the elements $z$ in the principal component. It is periodic and has for its primitive periods the elements $2 \pi i j$ where $j$ is any idempotent. These idempotents may be finite in number in which case one is dealing with a direct sum of irreducible rings; they may be denumerable or even nondenumerable. An example of the last is furnished by rings generated by suitable resolutions of the identity in reflexive spaces. This gives one a preliminary idea of the Riemann surface for $\log z$ which may have nondenumerably many sheets. In this connection the following extension of the Poincaré-Volterra theorem may be cited. Its proof is quite simple.

THEOREM 5. If a ring $\Re$ is separable, then any analytic function defined over a region in $\Re$ has at most denumerably many values.

It is not clear whether the same result applies to non-separable rings which are irreducible.

It is apparent from the example $w=\log z$ just considered that a periodic analytic function may have more than two periods; indeed it may have any number, finite or infinite, of periods. But if the ring is irreducible the picture becomes different. It seems probable that all primitive periods after the first two will lie in the radical. That this situation can arise may be shown by examples.

${ }^{21}$ As a special case, this terminology gives in the ring of complex numbers: $\sin 2$ is an analytic function of 2 !

${ }^{22}$ The first discussion of these components was given by Nagumo. See footnote 2 . 
For irreducible rings the notion of interiority to simple curves can be established by means of the integral (2) whose only possible values in this case are 0 and $e$. A simple curve $C: z=z(t), 0 \leqq t \leqq 1$, is one for which $t_{1} \neq t_{2}$ implies $\left[z\left(t_{1}\right)-z\left(t_{2}\right)\right]^{-1}$ exists. The interior of $C$ is then defined to consist of the points for which the integral equals $e$. It may be shown that interior and exterior points do exist. Although the interior and exterior are open sets they are not necessarily connected.

The question of ring extensions was raised in connection with reducibility. The question arises again in this form. If $a$ is singular in $\Re$ does there exist an extension $\Re_{1}$ of $\Re$ in which $a$ is regular? ${ }^{23}$ Further if $a$ is regular in $\Re_{1}$, does there exist an extension $\Re_{2}$ of $\Re_{1}$ in which $a$ is in the principal component (of the group of regular elements)? If the answer to this is affirmative, then $a$ may be embedded in a oneparameter group. This is a question "in the large." An alternative formulation of this problem is: Under what circumstances does $\log a$ exist in a ring extension?

Rings with one generator. Let $\Re$ be any normed ring and let $a$ be arbitrary in $\Re$. Consider the subset of $\Re$ consisting of the polynomials in $a$ (and $e$ ) and their limits. This set is a ring which is a subset of $\Re$ and will be designated by $\Re\{a\} .^{24} \mathrm{It}$ is clear that the spectrum of any element in $\Re\{a\}$ will include the spectrum of the same element considered as belonging to $\Re$. The expansion and shrinkage of spectra under ring contraction and extension is subject to certain laws. In particular, spectra which are purely real are absolute. ${ }^{25}$ From this point forward we shall treat exclusively rings $\Re\{a\}$.

The elements in $\Re\{a\}$ may properly be called functions of $a$. The nature of these functions will be examined. For example if $\Re$ is the ring of all continuous functions $f(x), 0 \leqq x \leqq 1$, with $|f|=1$.u.b. $[0,1]|f(x)|$, then by the Weierstrass approximation theorem, $\Re=\Re\{x\}$. The elements of $\Re\{x\}$ are in this case continuous functions of $x$. This and the examples 3,9 , and 10 introduced earlier indicate that each ring $\Re\{a\}$ defines a special type of function of $a$; in particular although every ring $\Re\{a\}$ contains analytic functions of $a$ (for example, $\exp (a), \sin a$ ), it does not except in special cases consist exclusively of such functions. It will be seen later that rings $\Re\{a\}$ are locally homomorphic to rings of types 3,10 , or 9 . Theorem 2 is an example of a homomorphism to a ring of continuous functions. Our

\footnotetext{
${ }^{23}$ A contribution to this problem has been made by G. Silov, On the extension of naximal ideals, C. R. (Doklady) Acad. Sci. URSS. vol. 29 (1940) pp. 83-84.

24 Observe that in the notation current in abstract algebra, $\mathfrak{R}[x]$ and $\mathfrak{R}(x)$ indicate extensions of $\Re$. For us here, $\Re\{a\}$ is a contraction of $\Re$.

${ }^{25}$ See footnote 12, Theorem 13.
} 
study may be considered as a reformulation and the first steps in a solution of the frequently considered problem of determining the analytic functions of an operator. The details of this undertaking will appear separately; in what follows, we shall give a brief review of the principal results. The problem may be recast in the following form: To determine conditions in order that a ring $\Re\{a\}$ should be homomorphic to a function ring of a given type. The types we shall discuss are (i) The functions analytic in a domain and continuous on its boundary; (ii) The functions infinitely differentiable on some interval. The case of functions of class $C^{m}, m \geqq 1$, can be elaborated on the basis of those of class $C^{\infty}$. The case of functions of class $C^{0}$ is handled by Theorem 2 .

The study of rings $\Re\{a\}$ may be considered as a study in polynomial approximation since all elements of $\Re\{a\}$ are limits of polynomials in $a$. The approximation is carried out over the spectrum of $a$. The norm for the approximation is at least as powerful as the uniform norm since $|p(a)| \geqq|p(\lambda)|$. The resolvent set of $a$ (the set complementary to the spectrum) is always connected.

If $a$ is a quasi-nilpotent then the ideal $(a)$ is the only maximal ideal in $\Re\{a\}$ and is identical with the radical. Examples show that $\Re\{a\}$ may have a radical even when $a$ is not a quasi-nilpotent.

Types of maximal ideals. We shall introduce a classification of maximal ideals in rings $\Re\{a\}$. This is an initial step in the study of the structure of the class of all ideals. For the rings treated here the maximal ideals are easily singled out. They are precisely the principal ideals $(a-\lambda e)$ where $\lambda$ is in the spectrum of $a$. Furthermore, Gelfand's topology of the class of maximal ideals coincides with the usual metric of the complex plane. If $a$ is singular then for any $n \geqq 1$ the ideal $\left(a^{n}\right)$ includes the ideal $\left(a^{n+1}\right)$. The inclusion may or may not be a proper one. In what follows, the inequality $\left(a^{n}\right) \neq\left(a^{n+1}\right)$ will indicate proper inclusion. Also, if for an integer $n,\left(a^{n}\right)=\left(a^{n+1}\right)$, then for all integers $r \geqq 1,\left(a^{n+r}\right)=\left(a^{n+r+1}\right)$. This leads us to our basic classification.

DEFinition. For the ideal (a), one of the three following cases will occur:

(i) $(a)=\left(a^{2}\right)=\cdots=\left(a^{n}\right)=\cdots \cdot$

(ii) $(a) \neq\left(a^{2}\right) \neq \cdots \neq\left(a^{n}\right)=\left(a^{n+1}\right)=\cdots$.

(iii) $(a) \neq\left(a^{2}\right) \neq \cdots \neq\left(a^{n}\right) \neq \cdots$.

The ideal (a) will be termed an ecto-, meso-, or endo-ideal according as it falls under case (i), (ii), or (iii). 
All three cases may arise. One may see that from a consideration of rings $\Re\{x\}$ in examples 2,9 , and 3 respectively. A more penetrating example of (i) is found in the ring $\Re\{H\}$ generated by a selfadjoint transformation in Hilbert space. Here the spectrum of $H$ is real and every ideal $(H-\lambda I)$ is an ecto-ideal.

By Theorem 2 the ring $\Re\{a\}$ is homomorphic to a ring of functions continuous over the spectrum of $a$. For ecto-ideals $(a-\lambda e)$, little more can be said. However if the ideals $(a-\lambda e)$ are meso- or endo-ideals the "functions" $f(a)$ are differentiable and may even be analytic. The precise facts will be stated below. We shall, however, give at this point a consequence of the endo-concept. Suppose that $(a)$ is an endo-ideal and that $f$ is in $\Re\{a\}$. Then for any integer $n$ we have a unique decomposition

$$
f=\beta_{0} e+\beta_{1} a+\cdots+\beta_{n} a^{n}+q_{n+1},
$$

where $q_{n+1} \in\left(a^{n+1}\right)$. This equation may be regarded as a form of Taylor theorem with remainder. It asserts that there exist projections of $\Re\{a\}$ on the ideals $(a),\left(a^{2}\right), \ldots$. These projections are the bounded linear transformations $T_{n}$ such that $T_{n} f=q_{n}$. Since $T_{n} q_{n}=q_{n}$, $T^{2}=T$.

It may be shown that if $\lambda$ is an interior point of the spectrum of $a$, then the ideal $(a-\lambda e)$ is an endo-ideal. The frontier points of the spectrum naturally call for delicate handling. Rather little is known about them at the present time.

A useful concept is that of a normed power series ring. Such a ring will consist of abstract power series which have been normed in some manner. In addition a ring of power series must contain all polynomials and convergence in the ring must imply the convergence of the coefficients of every power. Formula(5) asserts that the mapping $f \rightarrow\left\{\beta_{0}, \beta_{1}, \cdots\right\}$ transforms $\Re\{a\}$ into a ring of power series. In fact, if we set $\Im=\prod_{n=1}^{\infty}\left(a^{n}\right)$ the ring of power series is isomorphic topologically (by definition) and algebraically to the quotient ring $\Re\{a\} / \Im$.

Rings of power series are not limited to rings of analytic functions. Rings of infinitely differentiable functions, in particular quasianalytic rings, are of this type. In addition there exist rings which are the direct sum of a radical and the field of complex numbers, that is, rings which are almost entirely radical, which are power series rings. In these such series as $\sum_{n=0}^{\infty} n ! a^{n}$ may converge absolutely.

Rings of analytic functions. If $\lambda$ is any point in the spectrum of $a$, $(a-\lambda e)$ is a maximal ideal. Thus if $f \in \Re\{a\}$, by Theorem $2, f$ is mapped homomorphically on a complex valued function $f(\lambda)$ defined 
over the maximal ideals in $\Re\{a\}$, in this case, the spectrum of $a$. This function is continuous over the spectrum. For suppose $\left\{p_{n}(\lambda)\right\}$ is a sequence of polynomials such that $\lim _{n \rightarrow \infty} p_{n}(a)=f$. Then $p_{n}(a) \equiv p_{n}(\lambda) e \quad(a-\lambda e) ;$ and since $\left|p_{n}(a)-p_{m}(a)\right| \geqq\left|p_{n}(\lambda)-p_{m}(\lambda)\right|$ the convergence of $p_{n}(\lambda)$ to $f(\lambda)$ is uniform.

Suppose now that the spectrum of $a$ contains a neighborhood $D$ of the origin. Then $f(\lambda)$ is not merely continuous but also analytic in this neighborhood. A preliminary result is then that $\Re\{a\}$ is homomorphic to a ring of functions analytic in $D$ and continuous over the closure of $D$. Let us suppose that under this homomorphism $f \rightarrow f(z)$. It may be shown readily that the ideal $(a)$ is an endo-ideal; in fact every ideal $(a-\lambda e)$ where $\lambda \in D$ is an endo-ideal. Hence by equation (5) we may write

$$
f \sim \beta_{0} e+\beta_{1} a+\beta_{2} a^{2}+\cdots .
$$

The power series need not converge. (It is only in the ring $\Re\{a\} / \Im$ that a norm is defined for it.) A question which is raised immediately is: What is the relation between (6) and the Maclaurin expansion of $f(z)$ ? The answer is the expected one: The latter expansion is $f(z)=\beta_{0}+\beta_{1} z+\beta_{2} z^{2}+\cdots$. This fact is fundamental.

Now suppose that $D$ is the largest domain in the spectrum of $a$ which contains the origin. Consider for every $\lambda$ in $D$ the ideal $\Im_{\lambda}=\prod_{n=1}^{\infty}(a-\lambda e)^{n}$. Thus $\Im_{\lambda}$ consists precisely of all elements $f$ such that in the expansion (5) in powers of $(a-\lambda e)$, each $\beta_{i}$ is zero. Hence $f(z)=0$ in $D$. This means that $\Im_{\lambda}$ is independent of $\lambda, \Im_{\lambda}=\Im$. The principal theorem may now be stated.

TheOREM 6. Let $\Re\{a\}, D$, and $\Im$ satisfy the conditions given above. Then the ring $\Re\{a\} / \Im$ is isomorphic to a subring of the ring of functions analytic in $D$ and continuous over the closure of $D$. Under this isomorphism $a \rightarrow$. If $\left\{f_{n}\right\}$ is a sequence such that $\lim _{n \rightarrow \infty} f_{n}=f$, then $\lim _{n \rightarrow \infty} f_{n}(z)=f(z)$ uniformly in $D$.

This theorem states that $\Re\{a\}$ is locally (in the neighborhood of the origin) homomorphic to a ring of analytic functions. The norm dominating the ring of analytic functions is usually stronger than the ordinary uniform convergence norm. The ideal $\Im$ clearly contains the radical of $\Re\{a\}$. It also contains those elements generated by the connected components in the spectrum of $a$ which are distinct from the component containing $D$. It is also clear that in $\bar{\Re}=\Re\{a\} / \Im$ the element $\bar{a}$ has for its spectrum at least the closure of $D$. Whether it can contain more is not known. 
Rings of infinitely differentiable functions. The requirement that the spectrum of $a$ contain a neighborhood will be lightened. In this section will be considered certain rings $\Re\{a\}$ where $a$ has a real spectrum. More exactly the spectrum is to contain a certain real interval $[\alpha, \beta], \beta-\alpha>0$, and furthermore, for every $\lambda$ in this interval, the ideal $(a-\lambda e)$ is to be an endo-ideal. If $\left\{p_{n}(\lambda)\right\}$ is a sequence of polynomials such that $\lim _{n \rightarrow \infty} p_{n}(a)=f$ where $f$ is some element in $\Re\{a\}$, then for any integer $m$

$$
\begin{aligned}
p_{n}(a) \equiv & p_{n}(\lambda) e+p_{n}^{\prime}(\lambda)(a-\lambda e)+\cdots \\
& +p_{n}^{(m)}(\lambda)(a-\lambda e)^{m} \bmod (a-\lambda e)^{m+1} .
\end{aligned}
$$

Conditions can now be enforced to guarantee that not merely $\lim _{n \rightarrow \infty} p_{n}(\lambda)=f(\lambda)$ but that for every $m \geqq 1, \lim _{n \rightarrow \infty} p_{n}^{(m)}(\lambda)=f^{(m)}(\lambda)$. It was seen earlier that for every $\lambda$ and $m$ there exists a projection $\Re\{a\} \rightarrow(a-\lambda e)^{m}$. The condition that will be imposed is that the bound of this projection be a constant $M_{m}$ independent of $\lambda$ on $[\alpha, \beta]$. Finally let $\mathfrak{Q}$ be the ideal $\mathfrak{Q}=\prod_{m, \lambda}(a-\lambda e)^{m}$ where the intersection is formed for all $\lambda$ on $[\alpha, \beta]$ and all $m \geqq 1$. We are ready to state the principal result of this section.

TheOREM 7. Let $\Re\{a\},[\alpha, \beta]$, and $\mathfrak{Q}$ be as above. Let the projections $\Re\{a\} \rightarrow(a-\lambda e)^{m}$ be of bound $M_{m}$ independent of $\lambda$. Then the ring $\bar{\Re}\{a\}=\Re\{a\} / \mathcal{Q}$ is isomorphic to a subring of the ring of all functions infinitely differentiable on $[\alpha, \beta]$. The isomorphism carries a into $x$. If in $\Re\{a\}, \lim _{n \rightarrow \infty} f_{n}=f$ then uniformly on $[\alpha, \beta]$ and for $m \geqq 1$, $\lim _{n \rightarrow \infty} f_{n}^{(m)}(x)=f^{(m)}(x)$.

The condition of quasi-analyticity of a ring may be formulated in terms of ideals in a simple fashion. A ring of functions is quasianalytic on $[\alpha, \beta]$ if $\left.f^{(m)} \lambda\right)=0, m \geqq 0$, for some $\lambda \in[\alpha, \beta]$ implies $f(x)=0$ identically on $[\alpha, \beta]$. If we assume for the sake of simple notation that $\mathfrak{Q}$ in the above theorem is the zero ideal then we may say: $\Re\{a\}$ is quasi-analytic if and only if for some $\lambda$ on $[\alpha, \beta]$, $\Im_{\lambda}=\prod_{n=1}^{\infty}(a-\lambda e)^{n}=0$. It may be quickly verified that if $\Re\{a\}$ is quasi-analytic, it is a normed power series ring. This may be seen from formula (5) and the discussion which follows it.

Absolute values of real elements. The formula (2) is the principal one for determining the reducibility of rings. In the exploitation of this formula, care must be taken that the curve $C$ should not cross the spectrum of $a$. The question has been raised whether there are circumstances under which $C$ is allowed to cross the spectrum. We shall answer this in the affirmative, not indeed for the integrand in 
(2), but for a variation of it. Suppose for the sake of convenience that $a$ has a real spectrum which includes an interval around $\lambda=0$. Suppose further that for all real $\alpha \neq 0$, for some $M>0$, and some real $s<1,\left|a(\alpha i e-a)^{-1}\right| \leqq|\alpha|^{-s} M$. Then the following improper integral exists:

$$
b=\frac{1}{2 \pi i} \int_{c} \frac{a}{\zeta e-a} d \zeta,
$$

where $C$ is a simple rectifiable curve cutting the axis of reals orthogonally, once at $\lambda=0$, and once more far to the right. For example $C$ might be the circle $|\zeta-| a||=|a|$. It may be seen easily that the spectrum of $b$ is real and non-negative and that $b(a-b)=0$. In fact we may say that $b=\max (a, 0)$ and we may define the absolute value of $a$ by $|a|=2 b-a$.

The condition $\left|a(\alpha i e-a)^{-1}\right| \leqq|\alpha|^{-8} M$ implies that $(a)$ is an ecto-ideal. In fact the most frequently discussed cases of ecto-ideals seem to satisfy this inequality and hence lead to the notion of absolute value.

The use of the terminology $\max (a, 0)$ and $|a|$ may be justified as follows. According to Theorem 2, the element $a$ in $\Re\{a\}$ corresponds to the function $f(\lambda)=\lambda$. If $b$ corresponds to $g(\lambda)$, formula (8) modulo $(a-\lambda e)$ shows quickly that $b(\lambda)=\lambda$ if $\lambda \geqq 0, b(\lambda)=0$ if $\lambda<0$. Thus $b(\lambda)=\max (\lambda, 0)$. If we now assume that $\Re\{a\}$ has no radical, the correspondence of Theorem 2 is an isomorphism.

The principal ideals $(a-b)$ and $(b)$ have no common elements, their product is zero and together they span the maximal ideal $(a)$. In the cases usually considered they not only span $(a)$, but their direct sum is $(a)$.

These results may be used to prove the fundamental theorem concerning the structure of bounded self-adjoint transformations in Hilbert space. It is common knowledge that in establishing the equation

$$
H=\int \lambda d E(\lambda),
$$

no matter which of the many possible paths is taken, use has to be made at one or two critical points of powerful-if one prefers, subtletools whereas the remainder of the argument proceeds along more obvious lines. In the proof which we suggest these critical points are: (1) If $H$ is self-adjoint and its spectrum is contained within the real interval $[\alpha, \beta]$, then $|H|=\max (|\alpha|,|\beta|)$ (or alternatively, 
$\alpha \leqq(H f, f) \leqq \beta,(f, f)=1)$. (2) If $\gamma$ is an arbitrary point in $[\alpha, \beta]$ then there exist two self-adjoint transformations $H_{1}$ and $H_{2}$ in $\Re\{H\}$ such that the zero spaces of $H_{1}$ and $H_{2}$ form orthogonal manifolds whose direct sum is the Hilbert space $\mathfrak{S}$ and such that $H=H_{1}+H_{2}, H_{1} H_{2}=0$. The spectrum of $H_{1}$ lies essentially in $[\alpha, \gamma]$, that of $H_{2}$ in $[\gamma, \beta]$.

Proof of the first point is carried out with the help of an integral due to Hille which allows one to embed certain transformations with real non-negative spectrum in a semi-group. ${ }^{26}$ The only transformation we need is $H^{1 / 2}$ in case $\alpha \geqq 0$. The second difficulty is surmounted by means of formula ( 8 ) in the manner which we have indicated.

Barnard College, Columbia University

${ }^{26}$ See footnote 7, Theorem 14. 